\title{
Baikal-GVD: Time Calibrations in 2016
}

\author{
A.D. Avrorin ${ }^{a}$, A.V. Avrorin ${ }^{a}$, V.M. Aynutdinov ${ }^{a}$, R. Bannash ${ }^{g}$, I.A Belolaptikov ${ }^{b}$,
} V.B. Brudanin ${ }^{b}$, N.M. Budnev ${ }^{c}$, I.A. Danilchenko ${ }^{a}$, G.V. Domogatsky ${ }^{a}$, A.A. Doroshenko ${ }^{a}$, R. Dvornický ${ }^{b, h}$, A.N. Dyachok ${ }^{c}$, Zh.-A.M. Dzhilkibaev ${ }^{a}$, L. Fajt ${ }^{*}, h$, S.V Fialkovsky ${ }^{e}$, A.R. Gafarov ${ }^{c}$, K.V. Golubkov ${ }^{a}$, T.I. Gress ${ }^{c}$, Z. Hons ${ }^{i}$, K.G. Kebkal ${ }^{g}$, O.G. Kebkal ${ }^{g}$, M.M. Kolbin ${ }^{b}$, K.V. Konischev ${ }^{b}$, A.V. Korobchenko ${ }^{b}$, A.P. Koshechkin ${ }^{a}$, F.K. Koshel ${ }^{a}$, A.V. Kozhin ${ }^{d}$, V.F. Kulepov ${ }^{e}$, D.A. Kuleshov ${ }^{a}$, M.B. Milenin ${ }^{e}$, R.A. Mirgazov ${ }^{c}$, E.R. Osipova ${ }^{d}$, A.I. Panfilov ${ }^{a}$, L.V. Pan'kov ${ }^{c}$, D.P. Petukhov ${ }^{a}$ E.N. Pliskovsky ${ }^{b}$, M.I. Rozanov ${ }^{f}$, E.V. Rjabov ${ }^{c}$, G.B. Safronov ${ }^{b}$, B.A. Shaybonov ${ }^{b}$, M.D. Shelepov ${ }^{a}$, F. Šimkovic ${ }^{b, h, i}$, A.V. Skurikhin ${ }^{d}$, I. Štekl ${ }^{i}$, O.V. Surovovaa, V.A. Tabolenko ${ }^{c}$, B.A. Tarashansky ${ }^{c}$, S.A. Yakovlev ${ }^{g}$, A.V. Zagorodnikov ${ }^{c}$, and V.L. Zurbanov ${ }^{c}$

a Institute for Nuclear Research, Moscow, 117312 Russia

b Joint Institute for Nuclear Research, Dubna, 141980 Russia

c Irkutsk State University, Irkutsk, 664003 Russia

d Institute of Nuclear Physics, Moscow State University, Moscow, 119991 Russia

e Nizhni Novgorod State Technical University, Nizhni Novgorod, 603950 Russia

f St. Petersburg State Marine Technical University, St. Petersburg, 190008 Russia

g EvoLogics, Germany

h Comenius University, Mlynska Dolina F1, Bratislava, 84248 Slovakia

i Czech Technical University in Prague, Prague, 12800 Czech Republic

E-mail: lukas.fajteutef.cvut.cz

In April 2015, the first part (cluster) of the newly constructed next-generation neutrino telescope, Gigaton Volume Detector (GVD), was put into operation in the lake Baikal and started with data taking followed by another cluster installed in April 2017 thus doubling the number of installed Optical Modules (OMs) and instrumented volume. Moreover, the substantial extension of this detector is planned in the next few years. Specifically, another two clusters are going to be installed every consecutive year. In total 8 clusters consisting of $2304 \mathrm{OMs}$ with overall effective volume $\sim 0.4 \mathrm{~km}^{3}$ is going to be deployed by 2020 .

The vital condition for high angular resolution of reconstructed tracks of particles detected in GVD is a precise timing of individual channels which can be achieved with specialized time calibration systems. In this article, the different light sources and procedures used for time calibration of the GVD in the past as well as the newly developed ones are described and the results of the 2016 time calibration of the cluster Dubna are presented, especially the precision of intra and intersection calibrations and variations of calibration parameters in time.

35th International Cosmic Ray Conference - ICRC2017

10-20 July, 2017

Bexco, Busan, Korea

${ }^{*}$ Speaker. 


\section{Introduction}

The Gigaton Volume Detector (GVD) [1,2] is a neutrino telescope installed in the largest freshwater lake Baikal in Russia at $1366 \mathrm{~m}$ depth and $3.6 \mathrm{~km}$ from shore. The GVD is a threedimensional array of Optical Modules (OMs) arranged in larger units called clusters ( 1 cluster = 288 OMs). The first two clusters (576 OMs) were installed in April 2015 (called cluster Dubna) and April 2017 and are currently running. Two additional clusters are going to be deployed every next year up to 2020 when the whole detector consisting of eight clusters (2304 OMs) is going to be finished.

Every cluster consists of 8 strings ( 1 central +7 peripheral) of $525 \mathrm{~m}$ length separated by $60 \mathrm{~m}$. On every string there are $36 \mathrm{OMs}$ with $15 \mathrm{~m}$ spacing divided into 3 sections ( 1 section $=12 \mathrm{OMs}$ ). In every section, there is a Central Module (CeM) with 12-bit 12 channel $200 \mathrm{MHz}$ FADC responsible for digitization and processing of the pulses. In addition every string is equipped with 4 Acoustic Modules (AM) which measure exact position of the string in time and one Communication Module (CoM) which ensures the communication with a Cluster Center (CC).

The OM is a basic detection unit which contains a 10" PMT R7081-100, a controller, calibrations LEDs, an amplifier and a High Voltage (HV) converter, all enclosed in 17" pressure-resistant glass sphere. The main goal of the OMs is to detect the Cherenkov light emitted by charged particles created in Neutral Currents and Charged Current interactions of high energy astrophysical neutrinos.

The lake Baikal with its perfectly clear water provides ideal place for a neutrino telescope since the absorption and scattering are very low and the lake freezes for few months every year thus providing ideal platform for detector installation and maintenance. In 2016, the first cluster (Dubna) has taken 590 runs of 235 days of live time (77.7\% efficiency) and almost 1.8 billions of master events. In 2017, clusters work with $84.4 \%$ and $87.6 \%$ efficiency.

\section{Time Calibrations}

One of the main channels how to prove the existence of astrophysical neutrinos is a detection of high energy up-going muons created in charged current interactions of $v_{\mu}$. This channel provides the highest angular precision and thus enables the study of the point sources of emission. The crucial elements of the muon track reconstruction are the exact time of pulse detection and the actual position of OMs. The position is measured with AMs with very high precision $(\sim \mathrm{cm})$ every 40 seconds. To obtain the high precision in timing, the time calibration has to be performed.

The time calibration which measures the relative channel offsets (channel time delays) is in the GVD divided into three consecutive steps:

- Intra-section calibration: relative time delays between $12 \mathrm{OMs}$ in every section, two calibration methods $d T_{T S T}$ and $d T_{L E D}$

- Inter-section calibration: relatively between 24 sections in every cluster, two calibration methods Electronic and Muon runs

- Inter-cluster calibration: relatively between all installed clusters (= 2 in June 2017) 
The variations in channel offsets are caused mainly by differences in the cable lengths, PMT Transit Times (TT), and processing time of electronics. The goal of the calibration procedure is to measure channel offsets with a ns precision.

\section{Time Calibration Systems}

In order to verify obtained calibration parameters and estimate their precision, the GVD was designed with multiple calibration systems, which provide cross-check. In the following paragraphs, the brief description of GVD time calibration systems is given. ${ }^{1}$.

\section{Test Pulse}

In every OM, the test pulse can be created via the controller and its time of production can be adjusted with respect to LED light production. The test pulse is sent directly to amplifier (see Fig. 1a) while the light produced by LED is detected by PMT and produced photoelectrons have to propagate through system of dynodes which produce additional delay (TT). It means that the test pulses can be used to measure PMT's TT and also can be used as a reference time for measurements between neighbouring OMs.

\section{Built-in LEDs}

To be able to perform the basic intra and inter-section calibrations, every OM is equipped with two LED Kingbright L-7113PBC-A (see Fig. 1b) with peak wavelength $468 \mathrm{~nm}$ (blue), viewing angle 16 degrees, width $\sim 5 \mathrm{~ns}$ (FWHM) and adjustable intensity from $\sim 1$ up to $10^{8}$ photons.

\section{LED Matrices}

Every cluster contains 4-5 led matrices which can be used to perform inter-section calibration. A LED matrix contains 12 LEDs (same as in OMs) divided into two groups (6+6) controlled separately enclosed in 17" glass sphere (see Fig. 2a). In most cases, one group of LEDs is oriented vertically and the second one horizontally however also different arrangements of LEDs are tested. The light intensity of LEDs is adjustable in range $1-10^{8}$ photons and also the repeating period 0.1-1000 ms and time delay between individual LED groups 0-1000 ns can be set. The LED matrix can produce enough light to illuminate OMs further than $100 \mathrm{~m}$.

\section{Underwater Laser}

The newest time calibration system installed in April 2017 is a YAG: $\mathrm{Nd}^{3+}$ underwater laser (see Fig. 2b) which is going to be used for inter-cluster time calibrations. The peak wavelength is 532 $\mathrm{nm}$, the FWHM $=1 \mathrm{~ns}$ and the pulse energy $=0.37 \mathrm{~mJ}\left(\sim 10^{15}\right.$ photons $)$. The laser is controlled via ethernet by TCP protocol. The first measurements with the laser has shown its capability to illuminate OMs in both clusters and thus prove practicability of this new system.

\section{Time Calibrations in 2016}

The main goal of time calibrations in 2016 was to develop an automatized procedure of cluster time calibration and implement it to the BARS (Baikal Analysis and Reconstruction Software) [4] and in addition to use multiple time calibration systems to measure the accuracy of the obtained

\footnotetext{
${ }^{1}$ In April 2017 the POCAM (Precision Optical Calibration Module) [3] developed by IceCube collaboration for IceCube-Gen2 was installed in Dubna cluster for testing purposes.
} 


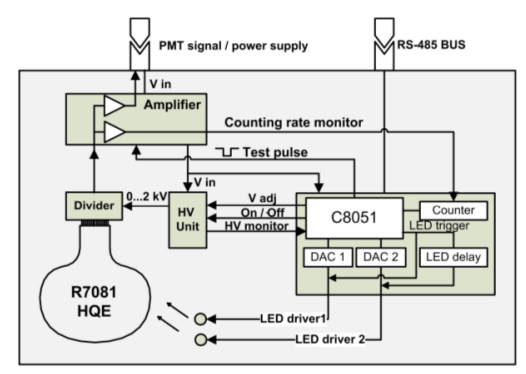

(a)

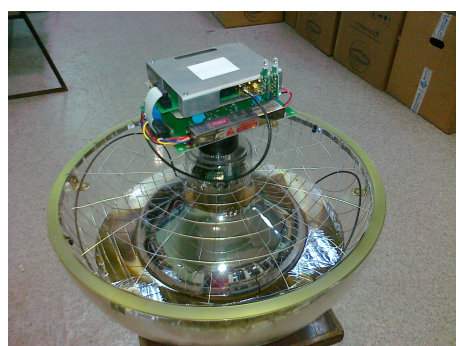

(b)

Figure 1: a) The block diagram of OM. b) The photo of internal parts of OM with two built-in LEDs in the upper part.

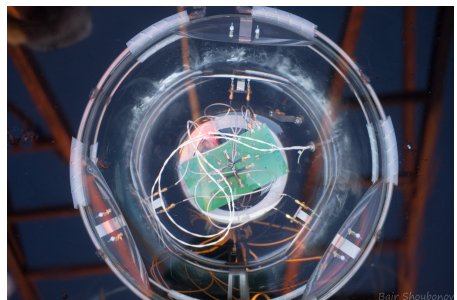

(a)

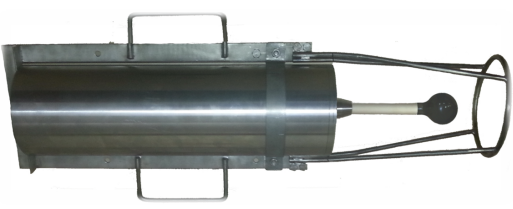

(b)

Figure 2: a) The photo of LED matrix. b) The visualization of underwater laser.

calibration parameters. In the following subsections, the intra and inter-section calibrations will be discussed in detail and the technique used for accuracy verification will be mentioned.

\subsection{Intra-section calibration}

In the intra-section calibrations the previous methods (discussed in [5]) were completely automatized, improved and implemented for all OMs in the cluster. The two methods for intra-section calibration called $d T_{T S T}$ and $d T_{L E D}$ both use built-in LEDs, however the calibration procedure is different and thus provide simple technique to compare results of time calibration and evaluate its precision. In $d T_{T S T}$ method the time delay $T_{T S T}^{i}$ of $i^{t h}$ channel is calculated as a sum of PMT TT and cable delay of $i^{t h}$ channel. The cable delays are measured on-shore and PMT TT is measured as a time difference between test pulse detection and LED light detection with both pulses produced in the OM controller in the same time. The relative time delay between two neighbouring channels can then be calculated as $d T_{T S T}^{i}=T_{T S T}^{i}-T_{T S T}^{i-1}$. The time evolution of PMT TT and therefore the time evolution of $T_{T S T}$ (since cable delays are constant due to constant operating conditions) was measured for more than 6 months. The results shown in Fig. 3a showing $T_{T S T 1}^{i}-T_{T S T 2}^{i}$ from two runs performed in April (2) and in October (1) indicate high stability (RMS $=0.27 \mathrm{ns)} \mathrm{of} T_{T S T}^{i}$.

In the $d T_{L E D}$ method the relative time delay between two neighbouring channels is measured as $d T_{L E D}^{i}=T o L P_{\text {meas }}^{i}-T o L P_{\text {expe }}^{i}$, where the ToLP means the Time of Light Propagation created in one OM and detected in the first upper OM. The distance between two OMs is known with high precision and time needed for light to propagate through a unit length is measured. It means that 


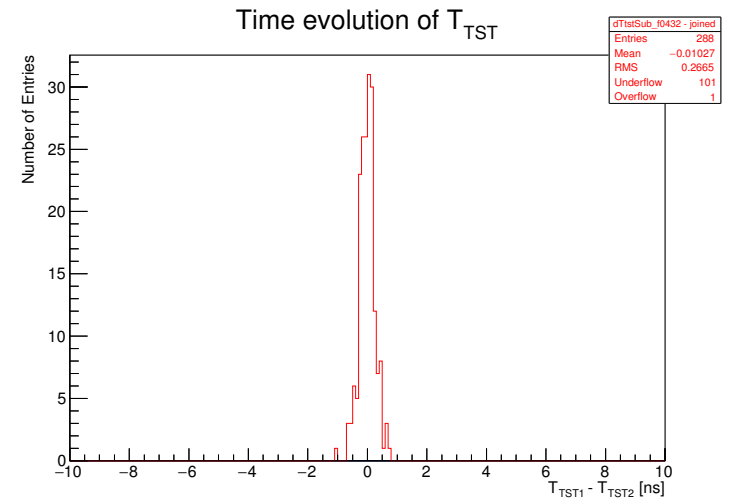

(a)

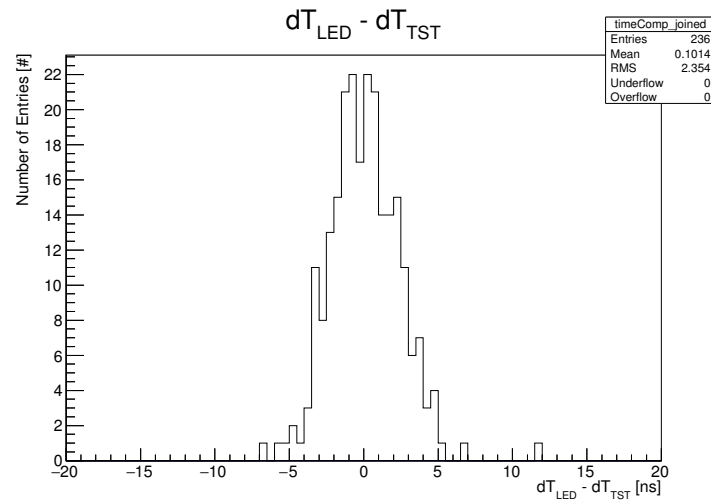

(b)

Figure 3: a) The time evolution of $T_{T S T}$ obtained from two different runs separated by 6 months. b) The distribution $\Delta T$ defined in text for all OMs showing the precision of the intra-section calibration.

$T o L P_{\text {expe }}$ can be measured with sufficient precision. All deviations from expected time of detection are explained as a relative time offsets between these two channels.

Both methods, $d T_{L E D}$ and $d T_{T S T}$ were used to obtain calibration parameters for all OMs in cluster Dubna. To study the accuracy of the obtained calibration parameters we define time residuals

$$
\Delta T^{i}=d T_{L E D}^{i}-d T_{T S T}^{i},
$$

which represent the time variance between our two calibration methods. The result of $\Delta T^{i}$ is shown in Fig. 3b. The mean of distribution is $0.10 \mathrm{~ns}$ and RMS, representing the uncertainty of the intrasection calibration, is $2.35 \mathrm{~ns}$.

\subsection{Inter-section calibration}

The inter-section calibration is used to measure relative delays between sections caused mainly by the differences in section cable lengths. Like in the intra-section calibration, two methods were used to measure calibration parameters and to obtain cross-check.

Both methods are based on the design of trigger system in GVD. The trigger request is created by $\mathrm{CeM}$ in the section in which the trigger condition is fulfilled. The trigger request is sent to $\mathrm{CC}$ through section cable. In the CC the trigger acknowledge signal is created, split and sent to CeM in every section. The signals producing trigger request are meanwhile stored in $5 \mu$ s FADC circular memory. When the trigger acknowledge arrives the stored pulses are sent through CC DAQ to the shore. From the position of the pulses in the FADC memory it is possible to measure the length of the section cables since the trigger request and acknowledgement are sent through this cable and meanwhile the pulses propagate through the memory.

The inter-section calibrations were measured with two methods, $d T_{\text {elec }}$ and $d T_{\text {part }}$. In the first method $d T_{\text {elec }}$, the PMTs are switched off and the artificial test pulses are used to create trigger request. In the second method $d T_{\text {part }}$, the triggers produced by real particles propagating through 


\begin{tabular}{lcc} 
Distribution & Mean & Stand. dev. \\
\hline \hline$d T_{\text {elec }}-d T_{\text {part }}$ & $4.12 \mathrm{~ns}$ & $2.72 \mathrm{~ns}$ \\
\hline$d T_{\text {part } 1}-d T_{\text {part } 2}$ & $-0.34 \mathrm{~ns}$ & $0.35 \mathrm{~ns}$ \\
\hline
\end{tabular}

Table 1: The results of inter-section calibration, namely comparison between two calibration methods and time evolution of inter-section time calibration parameters obtained from two runs.

the detector are used to study inter-section calibration parameters. The results of subtraction of calibration parameters obtained with these two methods (similar to $\Delta \mathrm{T}$ in the previous subsection) are shown in Tab. 1. Also the time evolution of calibration parameters obtained by $d T_{\text {part }}$ in two different runs is shown in the same table.

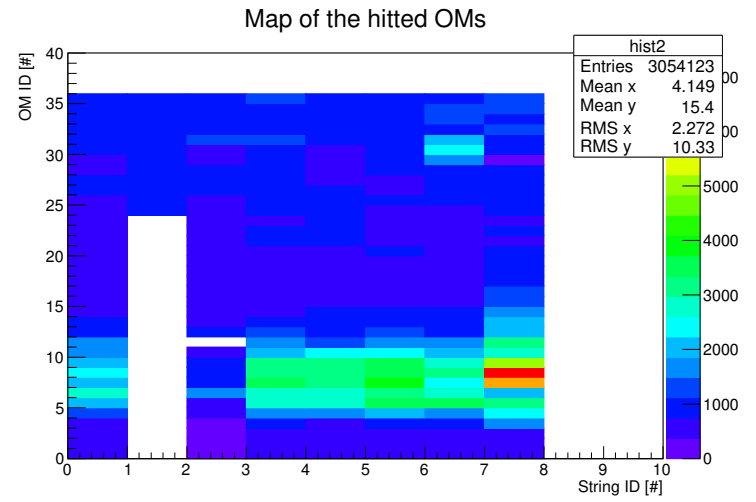

(a)

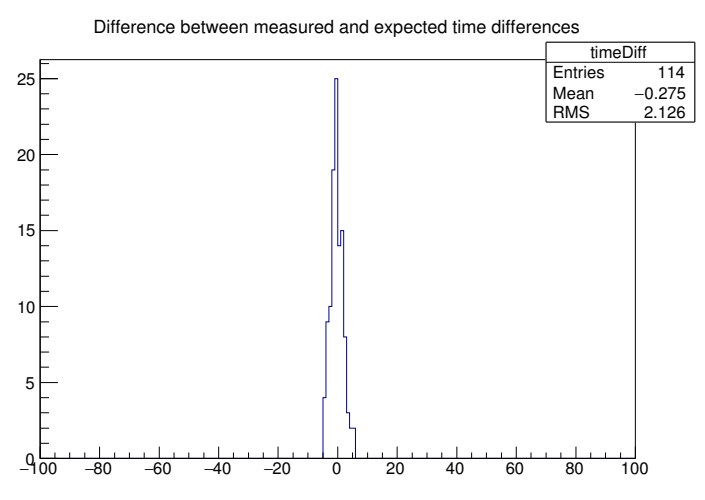

(b)

Figure 4: a) The map of OMs illuminated by LED matrix located at eighth string (string ID = 7) between 7th and 8th OMs. b) The distribution of differences between measured and expected time of detection of light produced in the LED matrix.

\subsection{LED matrix verification}

The final time calibration of the cluster is obtained combining the intra and inter-section calibration parameters. To verify its accuracy, the LED matrix runs are used. In the LED matrix run the LED matrix located at the central string produces light detectable in the sections at peripheral strings as well as in the upper neighbouring section (see Fig. 4a). The data obtained during these runs are calibrated with time calibration parameters and combined with information about current positions of OMs. Then the residuals between measured and expected time of light detection with respect to the real distance between LED matrix and individual OMs are calculated. The result of such a procedure is shown in Fig. 4b. The mean of the distribution is $-0.275 \mathrm{~ns}$ and the RMS representing the precision of the calibration is equal to $2.13 \mathrm{~ns}$.

\section{Conclusion}

In this article, the review of time calibration procedure and time calibration systems used in 
Gigaton Volume Detector was given. The time calibrations performed in 2016 are discussed in detail. The two methods for obtaining the intra-section as well as inter-section calibration were described. In both cases the cross-check reveals the calibration accuracy of approximately $2.5 \mathrm{~ns}$. In addition the time evolution of intra and inter-section calibration parameters were studied and results show low time dependence of $0.27 \mathrm{~ns}$ and $0.35 \mathrm{~ns}$ respectively. The final time calibration was validated by LED matrix runs.

\section{Acknowledgements}

This research is supported by the Grants of Comenius University in Bratislava, Slovakia UK/393/2016 and UK/398/2017, by the program OP VVV of the Czech Ministry of Education, Youth and Sports CZ.02.1.01/0.0/0.0/16_013/0001733 and by the Russian Foundation for Basic Research (Grants 16-29-13032, 17-02-01237).

\section{References}

[1] A.D. Avrorin et al., Baikal-GVD: first cluster Dubna, PoS EPS-HEP2015 418 (2015) [ins-det/151102324].

[2] A.D. Avrorin et al., The prototyping/early construction phase of the BAIKAL-GVD project, Nucl. Instrum. Meth. 742 (2014) 82-88 [astro-ph/13081833].

[3] M. Jurkovic et al., A Precision Optical Calibration Module (POCAM) for IceCube-Gen2, EPJ Web of Conferences 116 (2016).

[4] A.D. Avrorin et al., Data management and processing system for the Baikal-GVD telescope, this proceedings.

[5] A.D. Avrorin et al., LED based calibration systems of the Baikal-GVD neutrino telescope, EPJ Web of Conferences 116 (2016). 